\title{
Valganciclovir for the prophylaxis and treatment of cytomegalovirus infection in solid organ transplantation
}

This article was published in the following Dove Press journal:

Transplant Research and Risk Management

27 March 2010

Number of times this article has been viewed

\author{
Erin Wade Ticehurst' \\ Jennifer Trofe-Clark ${ }^{1,2}$ \\ Emily Blumberg ${ }^{3}$ \\ Roy D Bloom ${ }^{2}$
}

'Department of Pharmacy Services, Hospital of the University of Pennsylvania, Philadelphia, Pennsylvania, USA; ${ }^{2}$ Renal Electrolyte and Hypertension Division, Department of Medicine, University of Pennsylvania, Philadelphia, Pennsylvania, USA; ${ }^{3}$ Infectious Diseases Division, University of Pennsylvania, Philadelphia, Pennsylvania, USA
Correspondence: Erin Wade Ticehurst Department of Pharmacy Services, Hospital of the University of Pennsylvania, 3400 Spruce Street, Philadelphia, Pennsylvania 19104, USA

Tel $+|2| 5-6 \mid 5-8022$

Fax $+\mid$ 2I5-615-38|4

Email erin.ticehurst@uphs.upenn.edu
Abstract: Cytomegalovirus (CMV) can be a problematic virus for solid organ transplant recipients affecting both morbidity and mortality. Valganciclovir (VGC) is a commonly utilized antiviral agent for the prevention of this virus post-transplantation and recently it has been evaluated for the treatment of CMV. It is a pro-drug of ganciclovir (GCV) and has increased bioavailability compared to GCV. It is unclear whether VGC is superior to intravenous or oral GCV in terms of efficacy and safety in the prevention of CMV particularly in the liver transplant population as there have been studies reporting inferiority while other studies have not. Despite this, VGC has been reported to be the most commonly utilized agent for CMV prophylaxis in the liver transplant population in the United States and Canada. This article reviews CMV and VGC in the context of solid organ transplant, describes and assesses selected studies that have been conducted using this agent in this patient population, and summarizes VGC's advantages and disadvantages. Additional studies are needed to further define VGC's role in the treatment of CMV in the solid organ transplantation population as there are an insufficient number of studies pertaining to CMV treatment and no studies have been performed to assess its role in the treatment of life-threatening CMV disease. VGC is non-inferior to GCV for CMV prevention in the solid organ transplant population with the exception of liver transplant recipients.

Keywords: transplant, antiviral therapy, valganciclovir

\section{Introduction}

Cytomegalovirus (CMV), a commonly occurring virus in the general population, can have a negative impact on morbidity and mortality following solid organ transplantation. ${ }^{1,2}$ Although CMV infection is usually asymptomatic in the general population, ${ }^{3}$ the effects on the solid organ transplant recipient can be variable, ranging from asymptomatic viral shedding to fever and malaise, or symptomatic tissue-invasive disease. Indirect effects result from viral promotion of enhanced immunosuppression with subsequent development of other infections and posttransplant lymphoproliferative disorder and allograft rejection. ${ }^{1,4-7}$ This paper will review the use of valganciclovir (VGC) in the solid organ transplant population for the prevention and treatment of CMV infection and disease.

CMV is a member of the human herpesvirus family and the beta subfamily. ${ }^{8}$ The various modes of transmission include blood transfusions, sexual contact, transplacental, and direct contact with bodily secretions such as urine, breast milk, and saliva. ${ }^{3,9,10}$ Transplanted organs can transmit the virus from the donor to the recipient. ${ }^{10,11}$ Both the organ donor and the recipient of the transplanted organ are tested to determine 
CMV serostatus prior to transplantation by determining the presence of immunoglobulin $\mathrm{G}$ and $\mathrm{M}$ antibody to $\mathrm{CMV}$ which is consistent with prior infection. ${ }^{3,5}$

The course of CMV infection following transmission is largely affected by the level of competency of the host immune system. In the immunocompetent host, clinical infection or disease is rare following transmission however the virus typically remains latent in the host cells..$^{6,12,13}$ This is not the case in the solid organ transplant recipient however. As reported by Paya and colleagues, nearly half of all recipients who were seronegative prior to transplant and who received an organ from a seropositive donor will develop CMV viremia within 12 months of the transplant and in the majority of cases the onset of viremia occurs after antiviral prophylaxis is discontinued. ${ }^{14}$

CMV infection in the solid organ transplant recipient can be classified as primary infection, reactivation or superinfection. Primary infection refers to new transmission of the virus to a seronegative organ recipient $(\mathrm{R}-)$, either from a seropositive organ donor (D+), a blood transfusion, or a source within the community such as any of the modes of transmission described previously. Reactivation refers to a seropositive organ recipient's latent virus becoming active. Superinfection refers to a seropositive organ recipient becoming infected with a strain of CMV from the donor that is different than the strain they were latently infected with prior to transplant. ${ }^{5,15}$ Unfortunately, there are no standardized definitions for CMV infection or disease. Therefore, various definitions as well as terminology have been applied across clinical trials. In an effort to resolve this issue, in 2006 the American Society of Transplantation published proposed definitions for CMV infection and disease in organ transplant recipients for use in reporting infectious complications in immunosuppression clinical trials. ${ }^{16}$ The definitions are summarized in Table 1, and were modified from several sources ${ }^{5,15,17}$ including the American Society of Transplantation Infectious Disease Guidelines $^{5}$ and the Canadian Society of Transplantation consensus conference on $\mathrm{CMV}^{15}$

Table I American Society of Transplantation proposed definitions of CMV infection and disease in solid organ transplant recipients ${ }^{16}$

\begin{tabular}{|c|c|}
\hline Terminology & Definition \\
\hline CMV active infection & $\begin{array}{l}\text { Replicative infection that can be diagnosed by growing the virus in vitro, finding evidence of viral infection } \\
\text { by intra-cytoplasmic or intra-nuclear inclusions or by antibody-based staining techniques for CMV in } \\
\text { histopathologic sections or finding evidence of replication using nucleic acid based assays or antigenemia } \\
\text { studies. }\end{array}$ \\
\hline CMV disease & $\begin{array}{l}\text { Evidence of CMV infection with attributable symptoms. } \\
\text { Sub-divided into probable and definite CMV viral syndrome and tissue invasive disease, respectively. }\end{array}$ \\
\hline Probable CMV syndrome & $\begin{array}{l}\text { Characterized by one or more of the following: } \\
\text { I) Fever }>38^{\circ} \mathrm{C} \text { for at least } 2 \text { days } \\
\text { 2) New or increased malaise } \\
\text { 3) Leukopenia } \\
\text { 4) } \geq 5 \% \text { atypical lymphocytes } \\
\text { 5) Thrombocytopenia (threshhold not defined) } \\
\text { 6) Elevation of hepatic transaminases (ALT or AST) to } 2 \text { times the upper limit of normal (applicable to } \\
\text { nonliver transplant recipients) } \\
\text { Plus } \\
\text { Evidence of CMV in blood by viral culture, antigeniemia or a DNA/RNA based assay. }\end{array}$ \\
\hline Definite CMV syndrome & $\begin{array}{l}\text { Clinical and laboratory findings associated with probable CMV viral syndrome and no other cause of } \\
\text { symptoms/signs identified. }\end{array}$ \\
\hline Probable CMV tissue invasive disease & $\begin{array}{l}\text { The occurence of pneumonia, gastrointestinal disease, hepatitis, central nervous system disease, or } \\
\text { other sites (such as nephritis, cystitis, myocarditis and pancreatitis) } \\
\text { Plus } \\
\text { Evidence of CMV in the blood and/or tissue (detected by viral culture, antigeniemia, or a DNA/RNA } \\
\text { based assay), often in the absence of other documented causes. }\end{array}$ \\
\hline Definite CMV tissue invasive disease & $\begin{array}{l}\text { Clinical and laboratory findings associated with probable CMV tissue invasive disease } \\
\text { Plus } \\
\text { Detection of CMV in tissue by culture, immunohistochemical analysis or in situ hybridization. In addition } \\
\text { the presence of typical cytomegalovirus inclusions should be considered evidence of definite disease. }\end{array}$ \\
\hline
\end{tabular}

Note: CMV retinitis was not included as an applicable probable tissue invasive disease, but was classified as definitive disease if the lesions typical of CMV retinitis are confirmed by an opthalmologist.

Abbreviations: AST, aspartate aminotransferase;ALT, alanine aminotransferase; CMV, cytomegalovirus. 
The rate of CMV disease in organ recipients is influenced by many factors, including the pre-transplant donor/recipient serostatus, the immunosuppressive burden as well as the dose and duration of antiviral prophylaxis. In the era prior to routine prophylaxis, CMV disease occurred in $61 \%$ of renal transplant recipients. ${ }^{18}$ In the more contemporary era, rates of CMV disease of up to $50 \%$ have been reported following pancreas or kidney-pancreas transplantation and $25 \%$ following heart, liver, and kidney transplantation. ${ }^{7,19}$ The incidence of CMV disease following lung transplantation has been reported to be as high as $75 \% .{ }^{20}$ Rates of CMV infection and disease following solid organ transplantation vary per transplant center and this may be due to differences in immunosuppression management practices, viral monitoring and prophylaxis strategies. ${ }^{21}$

There are several risk factors for the development of CMV infection and disease in organ transplant recipients. Recipients who are CMV seronegative at the time of transplant and receive an organ from a seropositive donor are at higher risk for CMV infection than recipients who are CMV seropositive going into transplant. Other risk factors include blood transfusions, level of immunosuppression, and other host co-morbidities. ${ }^{5}$

The major purpose of antiviral prophylaxis therapy is to prevent viral reactivation and disease. It also shifts the onset of infection to later in the post-transplant period. This delay typically coincides with lower levels of immunosuppression at the time of infection for some patients, making the infection less severe and easier to treat because the host immune system is more active. Two types of CMV prevention strategies exist: pre-emptive therapy and universal prophylaxis. Preemptive therapy refers to initiating anti-CMV therapy when the viral load rises beyond a threshold value; ${ }^{5,15,22}$ this method relies on regular CMV viral load monitoring. Universal prophylaxis refers to giving antiviral therapy to all patients who are at risk of CMV regardless of the degree of risk. ${ }^{5,15,23}$

There are several methods that can be utilized for CMV surveillance. Two commonly used assays are nucleic acid testing (NAT) and CMV antigenemia. NAT may be reported as positive or negative (qualitative assay) or the viral deoxyribonucleic acid (DNA) can be quantified (quantitative assay). This assay offers both high specificity and high sensitivity and is useful in assessing the level of viral activity as well as response to therapy. ${ }^{22,24}$ Another test that can be helpful in identifying when a patient may require pre-emptive antiviral therapy is the antigenemia assay, an immunoassay that quantifies the amount of virus by measuring the number of white blood cells containing viral particles and offers moderate sensitivity and specificity. ${ }^{22}$
The antigenemia assay is less sensitive in patients with severe leukopenia as it requires the collection of a sufficient amount of peripheral blood neutrophils. ${ }^{25}$

\section{Valganciclovir Pharmacology}

VGC is a L-valyl ester prodrug of ganciclovir (GCV) that came to market in 2001 for CMV retinitis and in 2003 was approved in the United States by the Food and Drug Administration (FDA) for CMV disease prophylaxis in seronegative heart, kidney, and kidney-pancreas transplant recipients of organs from seropositive donors. ${ }^{26} \mathrm{VGC}$ is hydrolyzed into the active drug GCV and valine. ${ }^{2}$

To exert its anti-CMV effects, GCV must enter CMVinfected cells and be phosphorylated by the viral protein UL97 kinase. The product of this reaction is GCV monophosphate which is then phosphorylated by cellular kinases thus producing GCV diphosphate and finally GCV triphosphate which is an active inhibitor of DNA polymerase. . $^{2,27}$

UL97 kinase and/or viral DNA polymerase gene (UL54) mutations can impart GCV-resistance to CMV strains. ${ }^{28,29}$ Patient non-adherence and prolonged sub therapeutic ganciclovir exposure may contribute to the development of GCV-resistance. ${ }^{30}$ Two other antiviral agents (cidofovir and foscarnet) are options for treating UL97 kinase mutation driven GCV-resistant CMV disease as cross-resistance to these agents would not be expected. However, it should be noted that these two agents share an unfavorable adverse effect of nephrotoxicity. 5,31

\section{Pharmacokinetics}

GCV is the only known metabolite of VGC. ${ }^{23}$ Following oral administration with food the bioavailability of GCV from VGC is approximately $60 \%$ and systemic exposure to (VGC) is minimal. ${ }^{26,30,32}$ The GCV area under the curve (AUC) and the maximum concentration are increased when VGC is taken with food. An increased AUC has been associated with a decreased rate of infection; therefore VGC should be administered with meals. ${ }^{26,33}$

\section{Dosing}

The FDA-approved dose of VGC for the prevention of CMV disease in adult kidney, heart and kidney-pancreas transplant recipients with normal kidney function (creatinine clearance $>60 \mathrm{~mL} / \mathrm{min}$ ) is $900 \mathrm{mg}$ orally once daily which should be continued until 100 days post-transplant. 
Table 2 VGC dosing guidelines for patients with renal impairment ${ }^{26}$

\begin{tabular}{ll}
\hline Creatinine clearance (mL/min) & VGC dosing \\
\hline $40-59$ & $450 \mathrm{mg}$ orally daily \\
$25-39$ & $450 \mathrm{mg}$ orally every two days \\
$10-24$ & $450 \mathrm{mg}$ orally twice weekly \\
$<10$ or dialysis dependent & Not recommended \\
\hline
\end{tabular}

Abbreviation:VGC, oral valganciclovir.

VGC's metabolite (GCV) is excreted via the kidneys with a terminal half-life of $6.48 \pm 1.38$ hours in heart, kidney, kidney-pancreas and liver transplant recipients with normal renal function. ${ }^{26}$ Impaired renal function lengthens the half-life of GCV considerably. Therefore, VGC should be avoided in patients with a creatinine clearance $<10 \mathrm{~mL} /$ minute or those undergoing hemodialysis or peritoneal dialysis since appropriate dosage amounts and dose frequency have not been well-defined. ${ }^{26,34}$ Dosage adjustments are recommended for patients whose creatinine clearance is less than $60 \mathrm{~mL} /$ minute and the doses are described in Table 2. These doses are the FDA-approved doses for heart, kidney and kidney-pancreas recipients with renal insufficiency. A pharmacokinetic study conducted by Chamberlain and colleagues found that in the kidney transplant population a dose of VGC $450 \mathrm{mg}$ orally once daily provides similar systemic exposure to oral GCV $1000 \mathrm{mg}$ orally every 8 hours which was one of the more commonly used prophylaxis regimens prior to the availability of VGC but the exposure with a dose of $450 \mathrm{mg}$ of VGC is less than the exposure with $900 \mathrm{mg}$ of VGC. ${ }^{35}$

\section{Adverse effects}

The main adverse effects that affect the use of VGC in the solid organ transplant population are hematologic toxicities. These toxicities appear to be dose- and/or concentration dependent but may also be heightened by concomitant medications that solid organ transplant recipients commonly receive such as antiproliferative agents. The hematologic toxicities include anemia, thrombocytopenia, leukopenia, agranulocytosis and neutropenia. ${ }^{2,26,33}$

\section{Clinical trials}

Several prospective, randomized trials have evaluated VGC for CMV prophylaxis in solid organ transplant recipients, and one trial has evaluated VGC for treatment of non-life threatening CMV disease in this population. For the sake of brevity, only select clinical trials will herein be discussed in further detail. ${ }^{14,36-38}$ For a more comprehensive overview of VGC prophylaxis trials, the reader is referred to a systematic review which provides a review of all published studies up to April 2008 which assessed VGC as preemptive or prophylactic therapy in organ transplant recipients. ${ }^{39}$

\section{Pivotal trial of VGC for CMV prophylaxis in organ transplantation}

The pivotal trial supporting the FDA's approval of VGC as CMV prophylaxis in the organ transplant population compared VGC to oral GCV. GCV was the active comparator in this pivotal trial since it was already FDA approved for CMV prophylaxis in organ transplant recipients, and was considered the standard of care for CMV prophylaxis. ${ }^{14}$ Although the GCV pivotal trial ${ }^{40}$ had a matching placebo comparator when it was performed, due to the now proven efficacy of GCV, this design would have been unethical for the current VGC trial.

\section{Study design}

The study was designed as a prospective, multicenter, double-blind, and double-dummy study of CMV prophylaxis for high risk (CMV D+/R-) organ transplant recipients. Patients were stratified by allograft (kidney, liver, heart, kidney-pancreas) and randomly assigned in a 2:1 ratio at each center to receive VGC $900 \mathrm{mg}$ orally daily or oral GCV $1000 \mathrm{mg}$ three times a day within 10 days of transplant and through day 100 post-transplant. Quantitative CMV blood polymerase chain reaction (PCR) was monitored for up to 12 months post-transplant, and whenever CMV disease was suspected. Systemic exposure to GCV from either VGC or oral GCV was also assessed. The primary endpoint assessed the proportion of patients developing CMV disease (CMV syndrome and/or tissue invasive during the first six months post-transplant) as adjudicated by an independent (of both sponsor and study) blinded Endpoint Committee.

\section{Definitions of CMV disease}

Definitions of CMV syndrome and tissue invasive disease were similar to those summarized in Table 1. CMV syndrome was defined as fever $\geq 38^{\circ} \mathrm{C}$ on $\geq$ two occasions $\geq 24$ hours apart within a 7 day period, positive central laboratory results for CMV (measured by quantitative CMV PCR), and at least one of the following: new or increased malaise, two successive measurements of leukopenia (defined as white blood cell count of $<3500 \mu / \mathrm{L}$ or a white blood cell count decrease of $20 \%$ if the cell count prior to the development of clinical symptoms was $<4000 \mu / \mathrm{L}) \geq 24$ hours apart, atypical lymphocytosis $\geq 5 \%$, thrombocytopenia, or 
elevation of hepatic transaminases to $\geq$ twice the upper limit of normal (nonliver transplant recipients). Tissue invasive CMV was defined as symptoms or signs of organ dysfunction (excluding acute rejection in the grafted organ) and evidence of localized CMV infection in a biopsy or other appropriate specimen.

\section{Study results}

The intent to treat population consisted of 364 patients (239 received VGC and 125 received oral GCV), and were comparable in terms of demographics. The proportions of VGC versus oral GCV patients meeting the endpoint committee CMV disease definition were also comparable for each group (12.1\% versus $15.2 \%$ at 6 months and $17.2 \%$ versus $18.4 \%$ at 12 months respectively). VGC was associated with a 1.7 fold higher systemic exposure than oral GCV, and was consistent across allograft groups. Similar to what is observed in clinical practice, the majority of the cases of CMV disease occurred after discontinuation of prophylaxis. Adverse event profiles for both drugs were similar, though a non-significant higher incidence of neutropenia was seen with VGC $(8.2 \%)$ compared to oral GCV (3.2\%). Additionally, no GCV-resistant mutations were associated with VGC treated patients up to 1 year post-transplant. Although the overall incidence of CMV disease was comparable between the two treatment groups, statistical differences were noted in organ types, with liver transplant recipients treated with VGC experiencing a higher incidence of CMV disease.

In a subsequent FDA analysis of the liver transplant recipients in this study, there was both a higher rate of CMV disease (19\% versus 12\%) and tissue invasive disease (14\% versus $3 \%$ ) in the VGC group compared to GCV. This finding resulted in the FDA denying approval for VGC for use in liver transplant recipients. ${ }^{41}$

\section{Study significance and limitations}

This trial is distinguished as the pivotal trial that led to the FDA approval of VGC for CMV disease prophylaxis in CMV D+/R- heart, kidney and kidney-pancreas transplant recipients. Unlike the pivotal CMV prophylaxis trial with $\mathrm{GCV}^{40}$ the VGC trial compared two active comparators for CMV prophylaxis. Limitations of the study are that it did not include lung transplant recipients or all CMV serostatus combinations, and was designed as a non-inferiority study. It was also not powered to determine if improved bioavailability and efficacy of VGC would translate into better control of indirect CMV effects such as rejection, or improved compliance with once daily VGC dosing versus three times daily oral GCV dosing. Nevertheless, this trial represents the first clinical trial to demonstrate that VGC, a once daily dosed oral antiviral agent, has greater systemic exposure and similar efficacy and adverse event profile to GCV for CMV prophylaxis, with the exception of liver transplant recipients.

\section{VGC prophylaxis versus pre-emptive therapy in kidney transplantation}

Although VGC prophylaxis has been shown to decrease the incidence of CMV infection and disease, it is associated with significant drug costs as well as drug- toxicities. ${ }^{21}$ Therefore, a trial was performed to evaluate VGC prophylaxis compared to a pre-emptive approach for prevention of CMV infection and disease in kidney transplant recipients. ${ }^{36}$ The primary purpose of the study was to compare outcomes and cost associated with each method, which had not been previously done in a prospective, randomized fashion.

\section{Study design}

The study population consisted of adult kidney transplant recipients from a single center, and with all CMV donor/ recipient combinations except $\mathrm{D}-/ \mathrm{R}-$. Patients were randomized in a prospective 1:1 fashion and stratified by CMV status in an open label design to VGC $900 \mathrm{mg}$ orally daily for 100 days or preemptive treatment. Preemptive treatment consisted of initiating VGC $900 \mathrm{mg}$ orally twice daily for at least 21 days with the detection of CMV DNAemia (defined as $>2000$ copies/mL by blood PCR) and continued until CMV DNAemia was negative. Patients in either group who developed CMV infection received additional maintenance VGC $900 \mathrm{mg}$ daily for 3 months regardless of group assignment.

\section{Definition of CMV disease}

Published definitions of CMV infection were used. ${ }^{42}$ Of note, this same author's previously published work on CMV definitions ${ }^{17}$ contributed to the formation of the American Society of Transplantation CMV infection and disease definitions ${ }^{16}$ summarized in Table 1. CMV viremia was defined as the isolation of CMV by culture that involves the use of either standard or shell vial techniques. In contrast, CMV DNAemia was defined as the detection of DNA (qualitatively or quantitatively) in samples of plasma, whole blood, and isolated peripheral blood leukocytes or in buffy-coat specimens via PCR-based techniques, hybrid capture, and branchedchain DNA analysis. Asymptomatic CMV infection was 
defined as the detection of CMV DNAemia in the absence of symptoms. Symptomatic CMV infection was further divided into CMV syndrome and CMV target-organ diseases. The minimum requirements for $\mathrm{CMV}$ syndrome were defined as the documented presence of fever (temperature $>38^{\circ} \mathrm{C}$ ) for at least 2 days within a 4-day period, the presence of neutropenia or thrombocytopenia, and the detection of $\mathrm{CMV}$ in blood. (Note: quantitative values for neutropenia and thrombocytopenia were not given.) CMV target organ diseases were defined as the detection of CMV infection by culture, immunohistochemical analysis, or in situ hybridization together with histologic features of CMV on organ specific-biopsy.

\section{Study results}

Forty-nine patients were enrolled in each group respectively. CMV DNAemia occurred in $29 \%$ of patients in the prophylaxis group and $59 \%$ of patients in the preemptive group $(P=0.004)$. Median time of onset to CMV DNAemia was shorter for the pre-emptive group compared to the prophylaxis group [39 (range 14-81 days) versus 160.5 (range 10-307 days), $P=0.0002]$. Development of symptomatic CMV was similar between the groups, and was associated with mean higher peak levels of CMV DNAemia $(388,133$ copies $/ \mathrm{mL})$ versus asymptomatic infections (27,957 copies $/ \mathrm{mL}, P=0.001)$. Time to clearance did not differ between groups, but CMV serostatus was found to be an independent predictor of time to clearance. No clinical or virologic failure of VGC was evident to suggest resistance in patients with recurrent CMV DNAemia. Adverse event profiles, including leukopenia, were similar between groups. Overall costs were also similar between the groups. Using a cost of \$200 per CMV PCR test and \$24 per $450 \mathrm{mg}$ VGC tablet, the mean total cost per patient was $\$ 7130 \pm 3748$ for the preemptive group versus $\$ 7678 \pm 6486$ for the prophylaxis group $(P=0.611)$.

\section{Study significance and limitations}

Prior to this trial, prospective randomized trials to compare VGC prophylaxis with preemptive therapy for prevention of CMV infection and disease had not been performed. This study demonstrated that both prophylactic and preemptive therapies with VGC were associated with acceptable outcomes for CMV prevention. Moreover, it demonstrated that a preemptive approach to CMV prevention can also be safely utilized in a CMV D+/R-kidney transplant recipients. Overall costs were similar between strategies, though individual subcosts varied significantly between approaches. Limitations to this study are that it was open-label and only performed in a single center in a small number of kidney transplant recipients. It is unknown if these results are applicable to other organ transplant populations. The authors also hypothesized that there would be a $90 \%$ rate of CMV reactivation in the CMV $\mathrm{D}+/ \mathrm{R}$ - pre-emptive group. However, the overall incidences of CMV disease and CMV DNAemia in this subset were much lower than expected at $(7.7 \%$ and $53.8 \%$ at 12 months respectively), and was not entirely explained, though late onset disease CMV disease is less common with pre-emptive therapy. ${ }^{21}$ Finally, pre-emptive monitoring may not be as feasible for larger transplant centers and laboratory and drug costs will vary in different transplant practices.

\section{Meta-analysis of VGC versus other CMV prophylaxis regimens in organ transplantation}

Previous meta-analyses ${ }^{43,44}$ were unable to assess VGC to other CMV prophylaxis modalities, beyond the data from the prophylaxis pivotal trial. ${ }^{14} \mathrm{~A}$ recently published metaanalysis re-evaluated all experimental (randomized) and analytical (cohort and case control) studies that compared VGC with other therapies for CMV prophylaxis after all types of organ transplant until May 2008. ${ }^{45}$

\section{Study methods}

Nine studies ( 3 randomized, 3 cohort and 3 case control) consisting of 1831 patients met inclusion criteria. Seven trials comparing the efficacy of VGC to oral or intravenous GCV as prophylaxis in 1410 patients were included in the meta-analysis..$^{14,46-51}$ It was determined that 624 patients would be needed to provide a $90 \%$ power with a 2 -tailed alpha of $0.05 \%$ to detect a $7 \%$ reduction in CMV disease, and the meta-analysis was adequately powered. VGC comparator prophylaxis groups consisted of: IV GCV alone $\left(5 \mathrm{mg} / \mathrm{kg} /\right.$ day) in one study ${ }^{50}$ and GCV ( $3 \mathrm{~g} /$ day oral) in the other 6 studies ${ }^{14,46-49,51}$ Six trials also assessed the occurrence of neutropenia (defined as absolute neutrophil count $\left.<1500 / \mathrm{mm}^{3}\right) .{ }^{14,36,46,47,49,52}$ When comparing all included studies, prophylaxis ranged from 14 to 180 days and followup ranged from 6 to 12 months post-transplant.

\section{Definitions of CMV disease}

CMV disease was defined as the presence of CMV syndrome (viral detection with fever, malaise, or cytopenia) and/or end-organ disease involvement with CMV. CMV tissueinvasive disease was defined by the presence of end organ involvement by CMV. These definitions were based on the criteria defined by Ljungman et $\mathrm{al},{ }^{42}$ which were also used in 
the previous study reviewed ${ }^{36}$ and whose work contributed to American Society of Transplantation CMV infection and disease definitions ${ }^{16}$ summarized in Table 1. Late onset CMV disease was defined by the occurrence of CMV disease after completion of universal prophylaxis.

\section{Study results}

The overall risk for CMV disease did not differ significantly between the VGC and oral and IV GCV groups [odds ratio (OR) 0.98 (95\% confidence interval (CI) $0.67-1.43$; $P=0.92$; $\left.\left.\mathrm{I}^{2}=0 \%\right)\right]$. There was also no difference in the overall risk for late onset CMV disease (95\% CI 0.67-1.64, $P=0.84$; $\left.\mathrm{I}^{2}=0 \%\right)$. However, a separate analysis of two trials that compared VGC prophylaxis to acyclovir prophylaxis ${ }^{52}$ or pre-emptive therapy ${ }^{36}$ instead of GCV prophylaxis, found a statistically significant $795 \%$ higher rate of late onset CMV disease with VGC compared to acyclovir prophylaxis or preemptive therapy [OR 8.95 (95\% CI 1.07-74.83, $P=0.04$; $\left.\left.\mathrm{I}^{2}=0 \%\right)\right]$.

VGC increased the risk of neutropenia by $263 \%$ when compared to other preventative strategies [OR 3.63, (95\% CI 1.75-7.53; $\left.\left.P=0.001 ; \mathrm{I}^{2}=0 \%\right)\right]$, even in trials not using mycophenolate mofetil or induction therapy with $\mathrm{T}$ cell depleting therapies. Similar to the findings in the pivotal trial $^{14}$ and subsequent analysis ${ }^{41}$ this meta-analysis also found that in liver transplant recipients, the risk of CMV tissue invasive disease with VGC prophylaxis (14\%) was 4.5 times higher than with GCV (3\%) (95\% CI 1-20.14; $P=0.04)$ at 6 months, and 3.2 times higher with VGC (14\%) than with GCV (5\%) (95\% CI 0.99-11.19, $P=0.05)$ at 12 months. However, in the overall analysis, VGC was not found to be significantly associated with poorer survival.

\section{Study significance and limitations}

This study represents the first meta-analysis to compare VGC trials to other CMV preventative therapy, beyond the pivotal trial data. ${ }^{14}$ The analysis was adequately powered, found no publication bias, and showed no greater efficacy of VGC over IV and oral GCV. Similar to findings in previous studies, CMV tissue invasive disease was found to be significantly higher in liver transplant recipients. VGC was also associated with a significantly higher risk of absolute neutropenia. Additionally, it was noted that there was a higher incidence of late CMV disease with VGC compared to acyclovir or preemptive prophylaxis, but not when compared to GCV prophylaxis. Reasons for these findings are not completely understood, and the results were not limited to $\mathrm{CMV} \mathrm{D}+\mathrm{R}$ - recipients. A limitation of this meta-analysis was that it was not able to address the impact of the above mentioned adverse event on the post-transplant course. Other limitations outside of those inherent with this meta-analysis study design include the lack of data able to be presented on other opportunistic infections or patterns of CMV resistance to GCV. There were also no cost data analyzed among the various preventative strategies. Future studies may help to further delineate these issues.

\section{Treatment with VGC for CMV disease in organ transplantation}

To date, only one prospective randomized study has evaluated treatment of CMV disease in solid organ transplant recipients with VGC..$^{37}$ The primary outcome of the trial was treatment success defined as eradication of CMV viremia (eradication of CMV viremia was defined as $\leq 600$ copies $/ \mathrm{mL}$ of blood by CMV PCR) at day 21 post-treatment.

\section{Study methods}

The trial was a prospective, randomized 1:1, open-label, multicenter trial of adult solid organ transplant recipients with both virological and clinical evidence of non-life threatening CMV disease (regardless of CMV serostatus). Patients previously received anti-CMV therapy for prophylaxis, pre-emptive treatment or treatment of disease. In the intent to treat analysis, 164 patients were randomized to $900 \mathrm{mg}$ of VGC twice a day for 21 days, and 157 patients were randomized to IV GCV at a dose of $5 \mathrm{mg} / \mathrm{kg}$ twice a day for 21 days. Both groups received VGC $900 \mathrm{mg}$ orally once a day for days 22-49 and CMV blood PCR was monitored by a centrally blinded lab up to day 49 .

\section{Definition of CMV disease}

CMV disease was defined as the presence of CMV in the blood by a local assay including shell vial assay, antigenemia assay, or accredited nucleic acid detection assay, plus the presence of compatible symptoms. For viral syndrome the definition was consistent with current American Society of Transplantation recommendations for use in clinical trials (Table 1). ${ }^{16}$ Tissue invasive disease was defined as evidence of localized CMV infection in a biopsy or other appropriate specimen (such as bronchoalveolar lavage or cerebrospinal fluid) and/or relevant symptoms of organ dysfunction unlikely to be due to other causes.

\section{Study results}

Demographic and baseline characteristics were similar between the two groups, including the number of patients in each group. Clinical presentations, including the incidence of tissue invasive disease, baseline viral loads and patterns of 
decline in viral loads were also similar between the groups. Viral eradication was achieved in $45.1 \%$ of VGC and $48.4 \%$ of IV GCV patients at day $21(95 \% \mathrm{CI}-14 \%-8 \%)$ fulfilling non-inferiority criteria and at day 49 (67.1\% versus $70.1 \%$ respectively, $P=\mathrm{ns})$. The mean time to a clinically relevant drop ( $\geq 0.3$ natural $\log$ units) was $6.1 \pm 4.5$ days $(n=120)$ for VGC and $6.6 \pm 4.7$ days $(n=116)$ for IV GCV, $(P=n s)$. The median viral load half life and the median slope of viral load decay were comparable in both arms. Clinical resolution, as assessed by the investigators, occurred at a mean of 15.1 days for both groups $(P=\mathrm{ns})$. During the first 21 days, treatment discontinuations and frequency of adverse events were also similar between the groups. The only factor found to be predictive of viral eradication was the baseline viral load. The investigators noted that patients with a baseline viral load of $<10,000$ copies/mL had a univariate relative chance for eradication of viremia at day 21 of $6.41(95 \%$ CI 3.61-11.36, $P<0.001)$ and at day 49 of $2.56(95 \%$ CI 1.29-5.08, $P=0.001)$ compared to those with a viral load $\geq 10,000$ copies $/ \mathrm{mL}$. Despite clinical resolution, viral eradication was only achieved in approximately $58 \%$ of patients by day 21 . Therefore, the investigators recommend extension of therapy beyond 21 days, and individualization therapy based on viral load measurements.

\section{Study significance and limitations}

This study was the first prospective, randomized study to evaluate VGC for the treatment of CMV disease. There are several limitations to this study. The treatment arms compared oral to IV therapy and were not blinded; therefore, investigator bias may have been introduced. The study results can also only be applied to a subset of patients with CMV disease, as patients with life-threatening CMV illness were excluded. Moreover, no patient had extremely high viral loads (typically $>10^{6}$ ). The majority of patients (over 70\%) enrolled were kidney transplant recipients, and therefore it is not known if these results are applicable to other organ populations. Cost analysis, compliance with IV versus oral treatment regimens, and the impact of concurrent reduction of immunosuppression (not mandated for this study), were not assessed. Although this study represents the first randomized prospective trial to assess VGC as a treatment option for CMV disease, it was designed as a non-inferiority study. Future trials are necessary to further address these limitations.

\section{Long-term outcomes of CMV disease treatment with VGC versus IV GCV}

A subsequent 12-month follow-up study of the above mentioned study has also been published. ${ }^{38}$

\section{Study methods}

Patients assessed in this study were those from the above described study, ${ }^{37}$ who developed a positive CMV viral load measurement (central analysis) at the start of the study.

\section{Definition of CMV disease}

CMV disease recurrence was defined as CMV viral load $\geq 600$ copies $/ \mathrm{mL}$ occurring after the end of treatment, either at the scheduled (month 3 and 6) visits or upon evidence of clinical recurrence, in patients who had previously had two negative CMV viral loads by day 49 .

\section{Study results}

This study found that clinical and viral eradication as well as clinical and viral recurrences for CMV disease were similar between the groups. Opportunistic infections were also found to be similar between the groups. Additionally, during the treatment phase no differences in GCV resistant mutations were identified. In a multivariate analysis, the only independent predictor for recurrent CMV disease was viral eradication at day $21(P<0.0001)$. Therefore, weekly monitoring of viral loads until eradication is achieved is recommended. ${ }^{38}$

\section{Study significance and limitations}

This trial represents the long-term results of the first randomized trial comparing VGC to IV GCV for the treatment of CMV disease in organ transplant recipients. It further supports the findings of the initial report, showing that VGC is non inferior to IV GCV for the treatment of non-life-threatening $\mathrm{CMV}$ disease. It also helped us to gain more insight into the viral kinetics of CMV disease. Limitations to this trial are as previously stated in the first half of the trial. Moreover, no patient treated had life-threatening CMV disease, and hence it is unknown if the results would be applicable in this patient subset. Although this study represents the best evidence to date to assess VGC as a treatment option for CMV disease, future trials are necessary to further define its role in this setting.

\section{Discussion}

VGC has become a valuable treatment option in the prevention of CMV disease after organ transplant. Its therapeutic advantages and disadvantages in clinical practice are summarized below.

\section{Therapeutic advantages}

VGC is an accepted option for the prevention of CMV disease in the solid organ transplant population. Due to its increased 
bioavailability compared to oral GCV, VGC offers more convenient dosing (once daily compared to three times daily for prophylaxis). Its efficacy for CMV prophylaxis in many solid organ transplant populations is non-inferior to IV GCV so VGC offers the advantage of avoiding IV administration and the costs and risks associated with it (ie, line infections). The increased bioavailability may also lower the risk of the development of GCV-resistant CMV strains as it has the potential to decrease patient non-adherence while also increasing the drug level that the virus is exposed to compared to oral GCV.

\section{Therapeutic disadvantages}

VGC is still patent-protected so its cost for most institutions is likely higher than oral GCV which is not patent-protected. The necessity of dosage adjustments for patients with renal insufficiency is not ideal from a safety standpoint. There are potential drug interactions with VCG including that when used concomitantly with mycophenolate in patients with renal insufficiency exposure to both GCV and a metabolite of mycophenolate (mycophenolic acid glucuronide) may be increased. ${ }^{53}$ Other potential medications VGC may interact with include zidovudine, probenecid and didanosine. ${ }^{26}$

The hematologic toxicities associated with VGC are similar to several immunosuppressive medications commonly used in the solid organ transplant population and this can make the toxicities difficult to manage. Reducing the dosage of VGC to minimize the toxicity may increase the risk of developing GCV-resistant CMV strains.

The appropriateness of VGC use in the liver transplant population has been debated as various studies have reported clinical inferiority and non-inferiority compared to oral and IV GCV in this population. ${ }^{14,54,55}$ The FDA has not approved VGC for use in liver transplant recipients. ${ }^{26,41}$ Despite this, Levitsky and colleagues recently reported that VGC is commonly used in this patient population in the United States and Canada. ${ }^{56}$ Additional studies will be required to determine the efficacy of VGC for prevention and treatment of CMV in liver transplant recipients.

\section{Place in therapy}

The American Society of Transplantation and the Canadian Society of Transplantation both have published evidence based recommendations for CMV prevention and treatment. ${ }^{5,15}$ Briefly, these documents recommend oral VGC, oral GCV, or IV GCV for CMV prophylaxis in all solid organ transplant recipients at risk for CMV. When using VGC for CMV prophylaxis the recommended duration of therapy is three months. However, preliminary data in CMV high risk patients (CMV D+/R-) presented by Humar and colleagues at the 2009 American Transplant Congress suggests that these patients may benefit from a longer duration of prophylaxis. ${ }^{57}$

Likewise, the findings of Kalil and colleagues' metaanalysis support the American Society of Transplantation's recommendations. ${ }^{45}$ They report that the risk of CMV disease and overall efficacy are similar between VGC, oral and IV GCV despite the increased risk of CMV tissue invasive disease in liver transplant recipients and late onset CMV disease with VGC. Future studies are necessary to address the limitations identified from current clinical trials, and to further define the role of VGC as prophylaxis and treatment for $\mathrm{CMV}$ in solid organ transplant recipients.

\section{Disclosure}

Emily Blumberg receives research support and is a consultant for Roche and she also receives support from Viropharma.

\section{References}

1. Fishman JA. Infection in solid-organ transplant recipients. $N$ Engl $J$ Med. 2007;357(25):2601-2614.

2. Perrottet N, Decosterd LA, Meylan P, Pascual M, Biollaz J, Buclin T. Valganciclovir in adult solid organ transplant recipients: pharmacokinetic and pharmacodynamic characteristics and clinical interpretation of plasma concentration measurements. Clin Pharmacokinet. 2009;48(6):399-418.

3. Ho M. The history of cytomegalovirus and its diseases. Med Microbiol Immunol. 2008;197(2):65-73.

4. Mwintshi K, Brennan DC. Prevention and management of cytomegalovirus infection in solid-organ transplantation. Expert Rev Anti Infect Ther. 2007;5(2):295-304.

5. Cytomegalovirus. Am J Transplant. 2004;4 Suppl 10:S51-S58.

6. Freeman RB Jr. The 'indirect' effects of cytomegalovirus infection. Am J Transplant. 2009;9(11):2453-2458.

7. Rowshani AT, Bemelman FJ, van Leeuwen EM, van Lier RA, ten Berge IJ. Clinical and immunologic aspects of cytomegalovirus infection in solid organ transplant recipients. Transplantation. 2005;79(4):381-386.

8. Biron KK. Antiviral drugs for cytomegalovirus diseases. Antiviral Res. 2006;71(2-3):154-163.

9. Hassan J, Dooley S, Hall W. Immunological response to cytomegalovirus in congenitally infected neonates. Clin Exp Immunol. 2007;147(3):465-471.

10. McDevitt LM. Etiology and impact of cytomegalovirus disease on solid organ transplant recipients. Am J Health Syst Pharm. 2006; 63(19 Suppl 5):S3-S9.

11. Opelz G, Dohler B, Ruhenstroth A. Cytomegalovirus prophylaxis and graft outcome in solid organ transplantation: a collaborative transplant study report. Am J Transplant. 2004;4(6):928-936.

12. Froberg MK. Review: CMV escapes! Ann Clin Lab Sci. 2004;34(2): 123-130.

13. Griffiths PD, Cope AV, Hassan-Walker AF, Emery VC. Diagnostic approaches to cytomegalovirus infection in bone marrow and organ transplantation. Transpl Infect Dis. 1999;1(3):179-186.

14. Paya C, Humar A, Dominguez E, et al. Efficacy and safety of valganciclovir vs. oral ganciclovir for prevention of cytomegalovirus disease in solid organ transplant recipients. Am J Transplant. 2004;4(4):611-620. 
15. Preiksaitis JK, Brennan DC, Fishman J, Allen U. Canadian society of transplantation consensus workshop on cytomegalovirus management in solid organ transplantation final report. Am J Transplant. 2005;5(2):218-227.

16. Humar A, Michaels M. American Society of Transplantation recommendations for screening, monitoring and reporting of infectious complications in immunosuppression trials in recipients of organ transplantation. Am J Transplant. 2006;6(2):262-274

17. Ljungman P, Stanley PA. Workshop on cytomegalovirus disease; definitions, clinical severity scores and new syndromes. Scand J Infect Dis. 1995;99(Suppl):S87-S89.

18. Balfour HH Jr, Chace BA, Stapleton JT, Simmons RL, Fryd DS. A randomized, placebo-controlled trial of oral acyclovir for the prevention of cytomegalovirus disease in recipients of renal allografts. $N$ Engl J Med. 1989;320(21):1381-1387.

19. Peterson PK, Balfour HH Jr, Marker SC, Fryd DS, Howard RJ, Simmons RL. Cytomegalovirus disease in renal allograft recipients: a prospective study of the clinical features, risk factors and impact on renal transplantation. Medicine (Baltimore). 1980;59(4):283-300.

20. Speich R, van der Bij W. Epidemiology and management of infections after lung transplantation. Clin Infect Dis. 2001;33 Suppl 1: S58-S65.

21. Humar A, Snydman DR; and the AST Infectious Diseases Community of Practice. Cytomegalovirus in Solid Organ Transplant Recipients. Am J Transplant. 2009;9 Suppl 4:S78-S86.

22. Gerna G, Baldanti F, Torsellini M, et al. Evaluation of cytomegalovirus DNAaemia versus pp65-antigenaemia cutoff for guiding preemptive therapy in transplant recipients: a randomized study. Antivir Ther. 2007;12(1):63-72.

23. Baillie GM. Prevention of cytomegalovirus disease in solid organ transplant patients: prophylactic versus preemptive therapy. Am J Health Syst Pharm. 2006;63(19 Suppl 5):S10-S16.

24. Abu-Nader R, Patel R. Current management strategies for the treatment and prevention of cytomegalovirus infection in solid organ transplant recipients. BioDrugs. 2000;13(3):159-175.

25. Masaoka T, Hiraoka A, Ohta K, et al. Evaluation of the AMPLICOR CMV, COBAS AMPLICOR CMV monitor and antigenemia assay for cytomegalovirus disease. Jpn J Infect Dis. 2001;54(1):12-16.

26. Valcyte (valganciclovir) [package insert]. Nutley, NJ: Roche Laboratories Inc.; August 2009.

27. Crumpacker CS. Ganciclovir. N Engl J Med. 1996;335(10):721-729.

28. Boivin G, Goyette N, Rollag H, et al. Cytomegalovirus resistance in solid organ transplant recipients treated with intravenous ganciclovir or oral valganciclovir. Antivir Ther. 2009;14(5):697-704.

29. Limaye AP, Corey L, Koelle DM, Davis CL, Boeckh M. Emergence of ganciclovir-resistant cytomegalovirus disease among recipients of solid-organ transplants. Lancet. 2000;356(9230):645-649.

30. Baillie GM. Pharmacokinetics of antiviral agents for the treatment of cytomegalovirus infection. Am J Health Syst Pharm. 2005;62(8 Suppl 1): S14-S17.

31. Drew WL, Miner RC, Marousek GI, Chou S. Maribavir sensitivity of cytomegalovirus isolates resistant to ganciclovir, cidofovir or foscarnet. $J$ Clin Virol. 2006;37(2):124-127.

32. Brown F, Banken L, Saywell K, Arum I. Pharmacokinetics of valganciclovir and ganciclovir following multiple oral dosages of valganciclovir in HIV- and CMV-seropositive volunteers. Clin Pharmacokinet. 1999;37(2):167-176.

33. Wiltshire H, Paya CV, Pescovitz MD, et al. Pharmacodynamics of oral ganciclovir and valganciclovir in solid organ transplant recipients. Transplantation. 2005;79(11):1477-1483.

34. Czock D, Scholle C, Rasche FM, Schaarschmidt D, Keller F. Pharmacokinetics of valganciclovir and ganciclovir in renal impairment. Clin Pharmacol Ther. 2002;72(2):142-150.

35. Chamberlain CE, Penzak SR, Alfaro RM, et al. Pharmacokinetics of low and maintenance dose valganciclovir in kidney transplant recipients. Am J Transplant. 2008;8(6):1297-1302.
36. Khoury JA, Storch GA, Bohl DL, et al. Prophylactic versus preemptive oral valganciclovir for the management of cytomegalovirus infection in adult renal transplant recipients. Am J Transplant. 2006;6(9): 2134-2143.

37. Asberg A, Humar A, Rollag H, et al. Oral valganciclovir is noninferior to intravenous ganciclovir for the treatment of cytomegalovirus disease in solid organ transplant recipients. Am J Transplant. 2007;7(9):2106-2113.

38. Asberg A, Humar A, Jardine AG, et al. Long-term outcomes of CMV disease treatment with valganciclovir versus IV ganciclovir in solid organ transplant recipients. Am J Transplant. 2009;9(5):1205-1213.

39. Sun HY, Wagener MM, Singh N. Prevention of posttransplant cytomegalovirus disease and related outcomes with valganciclovir: a systematic review. Am J Transplant. 2008;8(10):2111-2118.

40. Gane E, Saliba F, Valdecasas GJ, et al. Randomised trial of efficacy and safety of oral ganciclovir in the prevention of cytomegalovirus disease in liver-transplant recipients. The Oral Ganciclovir International Transplantation Study Group [corrected]. Lancet. 1997;350(9093):1729-1733.

41. Lange W. Valcyte (valganciclovir $\mathrm{HCl}$ tablets) Dear Healthcare Professional Letter [Letter]. September 2003; Available at www.fda.gov. Accessed October 26, 2009.

42. Ljungman P, Griffiths P, Paya C. Definitions of cytomegalovirus infection and disease in transplant recipients. Clin Infect Dis. 2002;34(8):1094-1097.

43. Kalil AC, Levitsky J, Lyden E, Stoner J, Freifeld AG. Meta-analysis: the efficacy of strategies to prevent organ disease by cytomegalovirus in solid organ transplant recipients. Ann Intern Med. 2005;143(12):870-880.

44. Hodson EM, Craig JC, Strippoli GF, Webster AC. Antiviral medications for preventing cytomegalovirus disease in solid organ transplant recipients. Cochrane Database Syst Rev. 2008(2):CD003774.

45. Kalil AC, Freifeld AG, Lyden ER, Stoner JA. Valganciclovir for cytomegalovirus prevention in solid organ transplant patients: an evidence-based reassessment of safety and efficacy. PLoS One. 2009;4(5):1-10.

46. Akalin E, Sehgal V, Ames S, et al. Cytomegalovirus disease in high-risk transplant recipients despite ganciclovir or valganciclovir prophylaxis. Am J Transplant. 2003;3(6):731-735.

47. Humar A, Kumar D, Preiksaitis J, et al. A trial of valganciclovir prophylaxis for cytomegalovirus prevention in lung transplant recipients. Am J Transplant. 2005;5(6):1462-1468.

48. Keven K, Basu A, Tan HP, et al. Cytomegalovirus prophylaxis using oral ganciclovir or valganciclovir in kidney and pancreas-kidney transplantation under antibody preconditioning. Transplant Proc. 2004;36(10):3107-3112.

49. Park JM, Lake KD, Arenas JD, Fontana RJ. Efficacy and safety of low-dose valganciclovir in the prevention of cytomegalovirus disease in adult liver transplant recipients. Liver Transpl. 2006;12(1):112-116.

50. Said T, Nampoory MR, Pacsa AS, et al. Oral valgancyclovir versus intravenous gancyclovir for cytomegalovirus prophylaxis in kidney transplant recipients. Transplant Proc. 2007;39(4):997-999.

51. Weng FL, Patel AM, Wanchoo R, et al. Oral ganciclovir versus low-dose valganciclovir for prevention of cytomegalovirus disease in recipients of kidney and pancreas transplants. Transplantation. 2007;83(3):290-296.

52. Zamora MR, Nicolls MR, Hodges TN, et al. Following universal prophylaxis with intravenous ganciclovir and cytomegalovirus immune globulin, valganciclovir is safe and effective for prevention of CMV infection following lung transplantation. Am J Transplant. 2004;4(10):1635-1642.

53. MacPhee IA, Spreafico S, Bewick M, et al. Pharmacokinetics of mycophenolate mofetil in patients with end-stage renal failure. Kidney Int. 2000;57(3):1164-1168.

54. Pescovitz MD, Rabkin J, Merion RM, et al. Valganciclovir results in improved oral absorption of ganciclovir in liver transplant recipients. Antimicrob Agents Chemother. 2000;44(10):2811-2815. 
55. Shiley KT, Gasink LB, Barton TD, Pfeiffenberger P, Olthoff KM, Blumberg EA. Increased incidence of cytomegalovirus infection in high-risk liver transplant recipients receiving valganciclovir prophylaxis versus ganciclovir prophylaxis. Liver Transpl. 2009;15(8):963-967.

56. Levitsky J, Singh N, Wagener MM, Stosor V, Abecassis M, Ison MG. A survey of CMV prevention strategies after liver transplantation. Am J Transplant. 2008;8(1):158-161.
57. Humar A, Lebranch Y, Vincenti F, et al. The IMPACT study: Valganciclovir prophylaxis for until 200 days post-transpalnt in high risk kidney recipients substantially reduces the incidence of CMV disease. Am J Transplant. 2009;9(Issue s2), Abstract 201.

\section{Publish your work in this journal}

Transplant Research and Risk Management is an international, peerreviewed open access journal focusing on all aspects of transplantation and risk management to achieve optimal outcomes in the recipient improving survival and quality of life. The journal welcomes submitted papers covering original research, basic science, clinical studies, reviews \& evaluations, guidelines, expert opinion and commentary, case reports and extended reports. The manuscript management system is completely online and includes a very quick and fair peer-review system, which is all easy to use. Visit http://www.dovepress.com/ testimonials.php to read real quotes from published authors.

Submit your manuscript here: http://www.dovepress.com/transplant-research-and-risk-management-journal 